\title{
RETRACTED ARTICLE: An integrated parallel GNFS algorithm for integer factorization based on Linbox Montgomery's block Lanczos method over GF(2)
}

Laurence T. Yang • Li Xu • Jong Hyuk Park •

Sajid Hussain

Received: 20 October 2008 / Accepted: 6 February 2009

(C) Springer Science+Business Media, LLC 2009

This article has been retracted due to publishing someone's material without the knowledge of the author.

L. T. Yang · L. Xu (ه)

Department of Computer Science, St. Francis Xavier University, Antigonish, Canada

e-mail: txu@stfx.ca

L. T. Yang

e-mail: 1tyang@stfx.ca

J. H. Park

Department of Computer Science and Engineering, Kyungnam

University, Masan, Korea

e-mail: parkjonghyuk1@ @otmail.com

S. Hussain

Jodrey School of Computer Science, Acadia University, Wolfville,

Canada

e-mail: sajid.hussain@acadiau.ca 